\title{
Theory of Dissipationless Nernst Effects
}

\author{
Doron L. Bergman ${ }^{1}$ and Vadim Oganesyan ${ }^{2}$ \\ ${ }^{1}$ Department of Physics, California Institute of Technology, Pasadena, California 91125, USA \\ ${ }^{2}$ Department of Engineering Science and Physics, College of Staten Island, CUNY, Staten Island, New York 10314, USA
}

(Received 14 October 2009; published 11 February 2010)

\begin{abstract}
We develop a theory of transverse thermoelectric (Peltier) conductivity $\alpha_{x y}$, in a strong magnetic field - this particular conductivity is often the most important contribution to the Nernst thermopower. We demonstrate that $\alpha_{x y}$ of a free electron gas can be expressed purely and exactly as the entropy per carrier irrespective of temperature (which agrees with the seminal Hall bar result of Girvin and Jonson). In two dimensions we prove the universality of this result in the presence of disorder which allows explicit demonstration of a number of features of interest to experiments on graphene and other two-dimensional materials. We also exploit this relationship in the low-field regime and analyze the rich singularity structure in $\alpha_{x y}(B, T)$ in three dimensions; we discuss its possible experimental implications.
\end{abstract}

PACS numbers: 72.15.Jf, 72.20.Pa

The magnetothermoelectric Nernst-Ettingshausen effect [1] has enjoyed renewed interest in recent years, first as a probe of superconducting fluctuations, and more generally, as a novel transport characterization of electronic correlations. Following the initial work on the cuprates [2] strong magnetothermoelectricity was found in a variety of interesting materials. While precise theoretical treatment is lacking for most of these cases, phenomenological descriptions in terms of conventional weak-field quasiparticle transport theory [3-6] or effective classical hydrodynamic models $[7,8]$ have been used with varying degree of success [9].

In this Letter we break from these earlier studies to treat the finite field response directly, with no recourse to a lowfield regime. Our chief accomplishment is the exact expression of the off-diagonal Peltier conductivity $\alpha_{x y}$ in terms of entropy of free fermionic carriers (see Eqs. (5)(7) and (10). In two dimensions we prove the universality of this expression (which also applies to Dirac fermions) in the presence of quenched disorder and compare it against available experimental data. In three dimensions we obtain, essentially in a closed form, the entire intricate singularity structure in $\alpha_{x y}$ (as a function of magnetic field and temperature) inherited from the Landau level spectrum which bears strong resemblance to thermoelectric phenomenology of graphite [10]. As $\alpha_{x y}$ is somewhat of a less studied and, hence, poorly understood quantity, at least as compared to electrical conductivity or entropy, our basic result directly linking $\alpha_{x y}$ and entropy (importantly, without invoking the so-called "entropy currents" used elsewhere in the literature $[5,11]$ ) is useful both for simplifying computations but also for developing intuition.

Current flow in the presence of weak electric field and a small thermal gradient is determined by

$$
\begin{gathered}
\mathbf{J}=\sigma \cdot \mathbf{E}-\alpha \cdot \nabla T, \\
\mathbf{J}^{\mathbf{Q}}=T \alpha \cdot \mathbf{E}-\kappa \cdot \nabla T,
\end{gathered}
$$

where $\mathbf{J}, \mathbf{J}^{\mathbf{Q}}, T, \mathbf{E}, \sigma, \alpha, \kappa$ are, respectively, the charge and energy currents (slightly modulated in space), temperature and electric field strength, and electrical, Peltier, and heat conductivity tensors, respectively. Peltier conductivity is usually extracted from electrical conductivity and thermopower, $\mathbf{S}=\sigma^{-1} \cdot \alpha$, measured in a zero-current configuration.

For convenience we consider a particular, Hall "brick", sample shape (see Fig. 1) of finite extent along $x$ and $z$ axis, although our results will be independent of this assumption. Ignoring spin and possible valley quantum numbers the free electron Hamiltonian in a Landau gauge is

$$
\mathcal{H}=-\frac{\hbar^{2}}{2 m}\left[\left(\partial_{y}-x / \ell_{B}^{2}\right)^{2}+\left(\partial_{x}\right)^{2}\right]+\frac{\hbar^{2} k_{z}^{2}}{2 m}+V(x),
$$

where $m_{z}, m, V(x), B,-e$ and $\ell_{B}=\sqrt{\frac{\hbar}{e B}}$ are the electron masses in the $\hat{z}$ direction and $x$ - $y$ plane, confining potential along the $x$ axis, magnetic field along the $z$ axis, electron

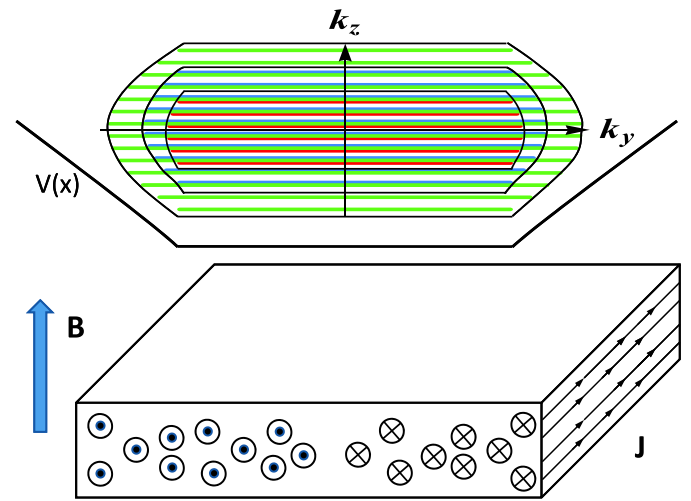

FIG. 1 (color online). Hall "brick" in a confining potential $V(x)$ (above) and its Fermi surfaces showing three occupied Landau bands (depicted using different colors). The spectrum is discrete along $k_{z}$ axis and continuous along $x$. The chiral surface states occupy nonflat portions of the Fermi surface and flow along brick's sides. 
charge and magnetic length, respectively. The confining potential along the $z$ axis (not shown explicitly) is assumed to be featureless (hard walls), whereas one along the $x$ axis, $V(x)$, has a more gradual rise as would be the case in graphite nanoribbons [12,13], for example. Consequently, the wave functions are standing waves along the $z$ axis, labeled with a discrete set of $k_{z}$ 's. If $V(x)=0$ the spectrum of this problem consists of Landau levels dispersing with $k_{z}, \epsilon_{n, k_{z}}=\hbar \omega_{n}+\frac{\hbar^{2} k_{z}^{2}}{2 m_{z}}$, where $\omega_{n}=\frac{e B}{m}\left(n+\nu_{0}\right)$, with $\nu_{0}=1 / 2$ (but can be different generally [14]). Provided $V(x)$ varies smoothly on scales set by the magnetic length, the spectrum of the Hall brick can be reconstructed by an adiabatic shift of Landau bands at each $k_{z}$ and $n$, e.g., by absorbing $V(x)$ into a spatially varying $\nu_{0}$. Thus, the Fermi sea of the Hall brick is a locus of points in the $k_{z}-k_{y}$ plane where $\epsilon_{n, k_{z}}<\mu$, the chemical potential (see Fig. 1). It is bounded by a set of closed Fermi surfaces each made up of two flat segments in the bulk (with $\mathbf{k}_{F, n}= \pm(\hat{z} / \hbar) \sqrt{\frac{\mu-\hbar \omega_{n}}{2 m_{z}}}$ ) and chiral surface states propagating in $\pm \hat{y}$ directions on opposite sides of the brick [15].

Bulk transport properties of the Hall brick can be computed using quantum Hall edge formalism $[16,17]$ for each transverse mode $k_{z}$. Recall $[16,17]$, that although microscopic current distributions depend sensitively on the details of the confining potential bulk transport coefficients computed by integrating over these distributions are independent of edge specifics, as they must be. The three nonzero Hall electric, Peltier, or thermal conductivities can be written as $\sigma_{x y}=-\frac{e^{2}}{h} C_{0}, \alpha_{x y}=\frac{k_{B} e}{h} C_{1}$ and $\kappa_{x y}=$ $-\frac{k_{B}^{2} T}{h} C_{2}$, respectively, with the help of $C_{q}=\sum_{k_{z}} c_{q}\left(k_{z}\right) / L_{z}$ and

$$
c_{q}\left(k_{z}\right)=-\sum_{n} \int_{\hbar \omega_{n}+\frac{\hbar^{2} k_{z}^{2}}{2 m_{z}}-\mu}^{\infty} d \epsilon\left(\frac{\epsilon}{k_{B} T}\right)^{q} \frac{\partial f(\epsilon)}{\partial \epsilon} .
$$

Here and elsewhere, the Fermi function is denoted by $f(\epsilon)=1 /\left(1+e^{\epsilon /\left(k_{B} T\right)}\right)$, with $k_{B}$ the Boltzmann constant. These coefficients are for electrons, for holes $\sigma_{x y}$ and $\kappa_{x y}$ reverse sign. Explicit expressions for $c_{q}$ can be obtained by a further variable change $d \epsilon \rightarrow d f$ and a definition $f_{n} \equiv$ $f\left(\hbar \omega_{n}+\frac{\hbar^{2} k_{z}}{2 m_{z}}-\mu\right)$, with a familiar result for $c_{0}\left(k_{z}\right)=$ $\sum_{n} f_{n}$ and somewhat less familiar expressions for $c_{1}\left(k_{z}\right)=$ $\sum_{n}\left[f_{n} \log f_{n}+\left(1-f_{n}\right) \log \left(1-f_{n}\right)\right] \quad$ and $\quad c_{2}\left(k_{z}\right)=$ $\sum_{n}\left[\frac{\pi^{2}}{3}+f_{n} \log ^{2}\left(1 / f_{n}-1\right)-\log ^{2}\left(1-f_{n}\right)-2 \operatorname{Li}_{2}\left(1-f_{n}\right)\right]$, where $\mathrm{Li}_{2}(z)$ is the polylogarithm function. Thus, we find that, up to an overall prefactor, $\alpha_{x y}$ is the entropy per particle added over Landau bands and averaged over transverse modes

$$
\alpha_{x y}=\frac{k_{B} e}{h L_{z}} \sum_{n} \sum_{k_{z}}\left[f_{n} \log f_{n}+\left(1-f_{n}\right) \log \left(1-f_{n}\right)\right] .
$$

Equation (5) is the basic result on which the rest of this Letter builds (thermal conductivity is left for future work [15]).
The two-dimensional limit of Eq. (5) is obtained by omitting the sum over $k_{z}$ modes and setting $f_{n}=$ $f\left(\hbar \omega_{n}-\mu\right)$

$$
\alpha_{x y}=\frac{e k_{B}}{h} \sum_{n=0}^{\infty}\left[f_{n} \log f_{n}+\left(1-f_{n}\right) \log \left(1-f_{n}\right)\right],
$$

which shows a sequence of thermally broadened nearly symmetric peaks of height $-\frac{k_{B} e}{h} \log 2$ as a function of $B$ or $\mu$, centered at quantum critical points separating integer Hall plateaux (where $\alpha_{x y}$ shows activated behavior). This structure was discovered and explored previously, albeit in the context of thermopower, by Girvin and Jonson [17,21] (although these authors apparently made no identification with the entropy). We now provide a proof that an exact quantum critical result in Eq. (6) is, in fact, universal in the presence of disorder.

For simplicity we consider a setting with generic, shortranged quenched disorder, strong magnetic field and no boundaries. We shall make use of two well-established facts about this system. First, we point out that the socalled "generalized" Mott formula

$$
C_{1}(T, \mu)=-\int_{-\infty}^{\infty} d \epsilon \frac{\partial f(\epsilon)}{\partial \epsilon} \frac{\epsilon}{k_{B} T} C_{0}(\mu+\epsilon, T=0)
$$

has been previously shown [21,22] to be an exact relation between $\sigma_{x y}(T=0)$ and $\alpha_{x y}(T)$. Second, it is now well established [23] that at $T=0$ the integer quantum Hall plateaus remain sharp steps in the presence of disorder, such that $\sigma_{x y}(T=0, \mu)=\frac{e^{2}}{h} \sum_{n} \theta\left(\mu-\mu_{C n}\right)$ [here $\theta(x<0)=0$ and unity otherwise]. The transitions are located at mobility edges $\mu_{C n}$ typically (but not necessarily) near the centers of clean Landau levels. Substituting the universal behavior of $\sigma_{x y}(T=0)$ into the generalized Mott formula we find a generalization of Eq. (6), with an important adjustment that $f_{n}$ should be evaluated at the mobility edges, $\mu=\mu_{C n}$, rather than the Landau levels of the problem without disorder.

Simple as it is, this proof is important on a number of counts. First, it corrects the findings of Jonson and Girvin [21], who studied the effects of quenched disorder using self-consistent Born approximation and concluded that their earlier, clean limit expressions [17] were not universal. Second, universality in the presence of disorder implies that any experimentally observed deviations must be due to experimental uncertainties, electron-electron or electronphonon interactions, disequilibration and/or finite size effects or other physics omitted thus far. Recent experiments on graphene [24] found a surprisingly robust confirmation of Eq. (6) for most plateaux transitions even in the strongly disordered samples, which also hints at the broader universality of Eq. (6), e.g., with respect to band structure details. In fact, we have confirmed this universality theoretically for Dirac fermions [15]. Third, the fact that the peaks are centered about the true critical points in the presence of disorder provides a very important additional 
insight highlighting the difference between the density of states (as measured through magnetization) and the entropy-while the former accounts for both localized and extended states, the entropy of localized states is strictly zero; hence, the activated behavior of $\alpha_{x y}$ is preserved. Fourth, the standard Mott formula

$$
\alpha(T)=-\left(\pi^{2} / 3\right)\left(k_{B}^{2} T / e\right) A_{\alpha} \partial \sigma(T, \mu) / \partial \mu,
$$

with $A_{\alpha}=1$ is violated in this dissipationless regime, essentially due to singularities of zero-temperature conductivity. For concreteness we can use (6) and the finite $T$ expression for $\sigma_{x y}$ in the absence of disorder to obtain $A_{\alpha}(\mu, T) \approx \frac{3}{\pi^{2}} \beta\left|\hbar \omega_{n}-\mu\right|$ in the activated regime and $A_{\alpha}=12 \log 2 / \pi^{2} \approx 0.84$ precisely at the transition-observe that Mott formula slightly overestimates $\alpha_{x y}$ near the transition and grossly underestimates it away, in the activated regime, as $T \rightarrow 0$. Finally, it is worth recalling that existence of delocalized states in two dimensions is due to a subtle interplay of magnetic field and disorder which becomes particularly intricate as $B \rightarrow 0$ [25]. Thus, while the generalized Mott relation continues to hold in this limit, the fate of $\sigma_{x y}$ (and $\alpha_{x y}$ ) rests with evolution of extended states, which may depend on factors not treated here, e.g., details of lattice structure [25]. In short, the limit $B \rightarrow 0$ is rather subtle and left for future work.

Next, we consider the three-dimensional regime of large $L_{z}$, so that a quasicontinuum of momenta $k_{z}$ appears below the Fermi level, so we can write simply

$$
\alpha_{x y}=\frac{e k_{B}}{h} \sum_{n} \int_{-\infty}^{\infty} \frac{d k_{z}}{2 \pi}\left[f_{n} \log f_{n}+\left(1-f_{n}\right) \log \left(1-f_{n}\right)\right] .
$$

One low temperature (Sommerfeld) expansion of this expression can be obtained by linearizing electron dispersion about each of the flat Fermi surfaces with Fermi velocity, $v_{F n}=\sqrt{2\left(\mu-\hbar \omega_{n}\right) / m_{z}}$

$$
\alpha_{x y}=-\frac{e k_{B}}{h} \frac{\pi^{2}}{3} \sum_{n=0}^{n_{\max }} \frac{k_{B} T}{2 \pi \hbar v_{F n}},
$$

where $n_{\max }=[\nu]$, the index of the highest occupied Landau level, is the integer part of $\nu=\left(\mu / \hbar \omega_{c}-\nu_{0}\right)$. Defining thermal de Broglie lengths for motion along the $z$ axis, $\lambda_{T z}=h / \sqrt{2 k_{B} T m_{z}}$ and perpendicular to it $\lambda_{T}=$ $h / \sqrt{2 k_{B} T m}$ we can rewrite

$$
\begin{aligned}
\alpha_{x y} & =-\frac{e k_{B}}{h} \frac{\pi^{2}}{3} \frac{\ell_{B}}{\lambda_{T z} \lambda_{T}} \sum_{n=0}^{n_{\max }} \frac{1}{\sqrt{\nu-n}} \\
& =i \frac{e k_{B}}{h} \frac{\ell_{B}}{\lambda_{T z} \lambda_{T}}\left[\xi(1 / 2,-\nu)-\xi\left(1 / 2,1+n_{\max }-\nu\right)\right]
\end{aligned}
$$

in terms of $\xi$, the Hurwitz zeta functions $(i \equiv \sqrt{-1})$. This approximate expression for $\alpha_{x y}$ vanishes linearly with $T$ as $T \rightarrow 0$, it also diverges at a set of critical fields $B_{C n}=$

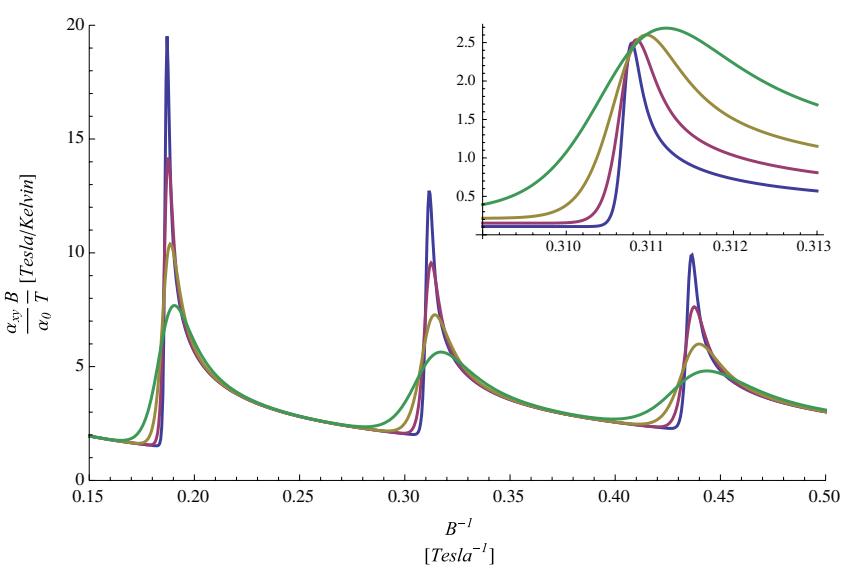

FIG. 2 (color online). Using effective mass parameters $m=$ $0.05 m_{e}, m_{z}=3 m_{e}$, and carrier density $n=10^{-18} / \mathrm{cm}^{3}$ we plot the expected behavior of $\alpha_{x y}$ across the quantum limit showing both $\sim T$ and quantum critical, $\sim \sqrt{T}$ (in the inset), behaviors. We take $T=0.5,1,2,4[K]$ for the $\sim T$ plot, and $T=$ $0.25,0.125,0.0625,0.03125[K]$ in the inset (identifiable by the fact that lower temperature gives sharper peaks). We compute $\alpha_{x y}$ in units of $\alpha_{0}=-\frac{k_{B} e}{h \lambda}$, where $\lambda=\frac{h}{\sqrt{2 m_{z} k_{B}(1 \text { Kelvin })}}$. The quantum critical behavior of the scaling function $F$ is characterized by a temperature and field independent value at the crossing point (signifying the crossing of the Landau band's bottom) and a temperature independent value at the peak $T$ away from the crossing point.

$\frac{\mu \mathrm{m}}{e\left(n+\nu_{0}\right)}$ defined by $\hbar \omega_{n}=\mu$ via one sided root singularities, due to one-dimensional van-Hove singularities appearing every time a Landau band empties, with $k_{F n} \rightarrow 0$. Clearly, Sommerfeld expansion breaks down around these critical points and we need to do better.

To that end we rewrite Eq. (9), with $b_{n} \equiv \beta\left(\mu-\hbar \omega_{n}\right)$ and $f_{z} \equiv f\left(z^{2}-b_{n}\right)$,

$$
\begin{gathered}
\alpha_{x y}=-\frac{e k_{B}}{h \lambda_{T z}} \sum_{n} F\left(b_{n}\right), \\
F(b) \equiv-\int_{-\infty}^{\infty} d z f_{z} \log f_{z}+\left(1-f_{z}\right) \log \left(1-f_{z}\right), \\
F(b)= \begin{cases}\sim 1 / \sqrt{b} & \text { for } b \rightarrow \infty \\
\sim e^{-b} & \text { for } b \rightarrow-\infty \\
\approx 2 & \text { at } b=0 \\
\approx 2.4 & \text { for } b=b_{\min } \approx 1.3\end{cases}
\end{gathered}
$$

in terms of the crossover function $F$ (which is everywhere positive), whose relevant regimes are listed above and displayed in Fig. 2. Most importantly, in quantum critical regimes $\hbar e\left|B-B_{C n}\right| / m \lesssim k_{B} T$ we predict $\sim \sqrt{T}$ variation of $\alpha_{x y} \approx-2(e / h)\left(k_{B} / \lambda_{T z}\right)$.

Experiments.-The broad range of materials where our theory may be tested directly includes conventional degenerate and nondengerate semiconductors [19], semiconductor heterostructures and graphene [24,26,27], bulk semimetals $[10,28]$, quantum critical points, esp. ones with pronounced changes in the Hall number [29]. Our discus- 
sion here will be limited to some very recent experiments on graphene and graphite- the relative abundance of data and features make these ideal test beds.

Several groups have recently examined thermoelectricity in graphene $[24,26,27]$ with data broadly confirming the existence of peaks in thermopower near plateaux transitions. In particular, analysis of the data to extract $\alpha_{x y}$ reveals [24] a reasonable agreement with Eq. (6), although the experimental values appear somewhat smaller than the theoretical prediction in the entire doping range studied. It is unclear at present whether the observed discrepancy is within experimental uncertainty or if additional theory of inelastic processes (e.g., electron-electron or electronphonon interactions) is necessary. As the noninteracting theory predicts peaks whose heights are independent of temperature, with widths (in field or gate voltage) narrowing as $T \rightarrow 0$ it would be of particular interest to measure the temperature dependence of $\alpha_{x y}$.

Our theory for three dimensions appears to capture gross experimental features in graphite [10] rather well. The overall scale of the observed signal is reproduced by the effective mass parameters for two dominant electron and hole bands previously used [30] in graphite: the estimate of $\alpha_{x y}$ was obtained from thermopower data in Ref. [10] by taking diagonal resistivity $\rho(B) \approx 2 \times 10^{-5} B \Omega \cdot \mathrm{m}$ per Tesla and neglecting $\alpha_{x x} \rho_{x y}$ contribution. Most importantly, the experiments clearly demonstrate the existence of asymmetric singularities synchronous with Landau level emptying transitions. Well away from these transitions $\alpha_{x y}$ usually vanishes linearly with temperature, as expected by the simple Sommerfeld expansion of Eq. (10). While showing a clear breakdown of $\sim T$ scaling near the singularities, the existing data [10] are insufficient to confirm the $\sim \sqrt{T}$ law discussed above, unfortunately.

In conclusion, we identified carrier entropy with $\alpha_{x y}$ exactly, irrespective of temperature. We demonstrated that strong localization in two dimensions enforces this relationship, computed $\alpha_{x y}$ in three dimensions and examined available experimental data in graphene and graphite. While there is considerable degree of qualitative and quantitative agreement between our theory and the experiments, much work remains to appreciate the full reach and importance of our observation. Clearly, some theoretical treatment of inelastic processes is needed-while it appears [15] that weak electron-electron interactions leave our result intact, phonon effects (e.g., drag) on the Nernst thermopower are less understood [18,31]. Careful direct measurements (or extractions) of $\alpha_{x y}$ will be of great use as well. Finally, strongly correlated regimes, both at zero [32] and finite [33,34] temperatures may also exhibit a version of $\alpha_{x y}$-to-entropy correspondence.

We would like to thank K. Behnia, S. Girvin, L. Glazman, and P. Ong for useful discussions. We are especially grateful to K. Behnia for sharing with us his unpublished results and reigniting our interest in Nernst matters. This work was supported by the National Science
Foundation through Grant No. DMR-0803200 and through the Yale Center for Quantum Information Physics.

[1] J. Ziman, Electrons and Phonons: The Theory of Transport Phenomena in Solids (Oxford University Press, USA, 2001).

[2] Z. Xu, N. Ong, Y. Wang, T. Kakeshita, and S. Uchida, Nature (London) 406, 486 (2000).

[3] V. Oganesyan and I. Ussishkin, Phys. Rev. B 70, 054503 (2004).

[4] A. Hackl and S. Sachdev, Phys. Rev. B 79, 235124 (2009).

[5] C. Zhang, S. Tewari, V. Yakovenko, and S. Das Sarma, Phys. Rev. B 78, 174508 (2008).

[6] S. Tewari and C. Zhang, Phys. Rev. Lett. 103, 077001 (2009).

[7] I. Ussishkin, S. Sondhi, and D. Huse, Phys. Rev. Lett. 89, 287001 (2002).

[8] D. Podolsky, S. Raghu, and A. Vishwanath, Phys. Rev. Lett. 99, 117004 (2007).

[9] K. Behnia, J. Phys. Condens. Matter 21, 113101 (2009).

[10] Z. Zhu, H. Yang, B. Fauque, Y. Kopelevich, and K. Behnia, arXiv:0909.2137.

[11] N. Cooper, B. Halperin, and I. Ruzin, Phys. Rev. B 55, 2344 (1997).

[12] S. Sun and C. Chang, Eur. Phys. J. B 64, 249 (2008).

[13] K. Novoselov, E. McCann, S. Morozov, V. Fal'ko, M. Katsnelson, U. Zeitler, D. Jiang, F. Schedin, and A. Geim, Nature Phys. 2, 177 (2006).

[14] L. Smrcka and N. Goncharuk, arXiv:0904.4114.

[15] D. Bergman and V. Oganesyan (unpublished).

[16] B. Halperin, Phys. Rev. B 25, 2185 (1982).

[17] S. Girvin and M. Jonson, J. Phys. C 15, L1147 (1982).

[18] P. Zyryanov and G. Guseva, Phys. Usp. 11, 538 (1969).

[19] R. Fletcher, Semicond. Sci. Technol. 14, R1 (1999).

[20] W. Wu, N. Ong, and P. Chaikin, Phys. Rev. B 72, 235116 (2005).

[21] M. Jonson and S. Girvin, Phys. Rev. B 29, 1939 (1984).

[22] L. Smrcka and P. Streda, J. Phys. C 10, 2153 (1977).

[23] B. Huckestein, Rev. Mod. Phys. 67, 357 (1995).

[24] J. G. Checkelsky and N. P. Ong, Phys. Rev. B 80, 081413 (2009).

[25] B. Huckestein, Phys. Rev. Lett. 84, 3141 (2000).

[26] P. Wei, W. Bao, Y. Pu, C. Lau, and J. Shi, Phys. Rev. Lett. 102, 166808 (2009).

[27] Y. M. Zuev, W. Chang, and P. Kim, Phys. Rev. Lett. 102, 096807 (2009).

[28] K. Behnia, M. Méasson, and Y. Kopelevich, Phys. Rev. Lett. 98, 166602 (2007).

[29] S. Paschen, T. Lühmann, S. Wirth, P. Gegenwart, O. Trovarelli, C. Geibel, F. Steglich, P. Coleman, and Q. Si, Nature (London) 432, 881 (2004).

[30] X. Du, S. Tsai, D. Maslov, and A. Hebard, Phys. Rev. Lett. 94, 166601 (2005).

[31] M. Matsuo et al., Phys. Rev. B 80, 075313 (2009).

[32] K. Yang and B. Halperin, Phys. Rev. B 79, 115317 (2009).

[33] Y. Wang, Z. Xu, T. Kakeshita, S. Uchida, S. Ono, Y. Ando, and N. Ong, Phys. Rev. B 64, 224519 (2001).

[34] O. Cyr-Choinière et al., Nature (London) 458, 743 (2009). 